\title{
Rehabilitación del paciente edéntulo con la técnica all on four mediante prótesis implanto-soportada: Reporte de caso.
}

Rehabilitation of the edentulous patient with the art all on four implant-supported prostheses by: Case report.

Dulio Maravi Escurra ${ }^{1, a}$, Antonio Balarezo Razzeto ${ }^{2, b, c}$, Eduardo Vicente Zamudio $^{2, b, c}$

\section{RESUMEN}

El desarrollo de los implantes dentales revoluciono las posibilidades de rehabilitación de pacientes que requieren la sustitución de dientes. La colocación de implantes dentales inmediatos post-exodoncia es un procedimiento quirúrgico alternativo al protocolo original de Branemark que tiene como principal ventaja evitar la modificación irreversible de los tejidos bucales debido al proceso fisiológico de reabsorción. El objetivo de este trabajo es relatar un caso clínico en el cual se realizaron exodoncias de dientes de pronostico dudoso y sustituidos por una rehabilitación implanto soportada mandibular utilizando el concepto All on Four.

\section{Palabras clave: IMPLANTES DENTALES, ALL ON FOUR, REHABILITACIÓN IMPLANTO SOPORTADA}

\section{SUMMARY}

The development of dental implants has revolutionized the possibilities of oral rehabilitation in patients requiring teeth replacement. The inmediate placement of dental implants post-extraction is a surgical procedure alternative to the original Branemark protocol whose main advantage avoid irreversible change of the oral tissues because the physiological process of reabsorption. The aim of this study is to report a case in which tooth extractions performed doubtful prognosis and replaced by implants supported mandibular rehabilitation using the concept All on Four.

\section{Keywords: DENTAL IMPLANTS, ALL ON FOUR, IMPLANT-SUPPORTED REHABILITATION}

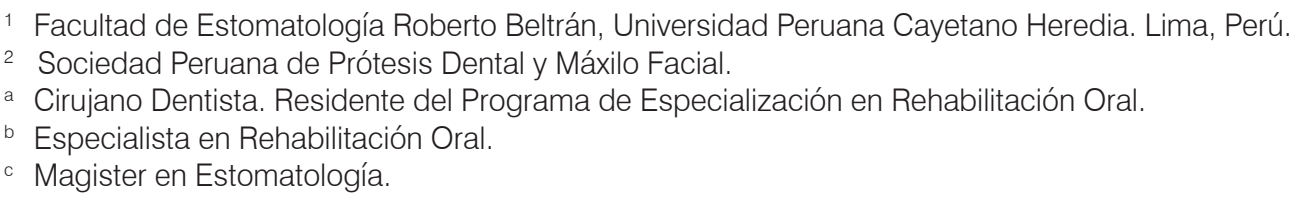




\section{INTRODUCCIÓN}

Los implantes dentales se han convertido en técnicas terapéuticas predecibles, basados en el protocolo de Bränemark (1), durante más de 44 años, gracias a sus estudios y tasas de éxito a lo largo de este periodo, durante el cual se han logrado reponer dientes perdidos o ausentes en pacientes adultos.

La técnica all-on-four (all-on-4), desarrollada por el Dr. Paulo Maló, (2), es un sistema que permite la rehabilitación total fija con implantes del maxilar superior $\mathrm{y} / \mathrm{o}$ inferior en el paciente desdentado total.

Su nombre procede de la utilización de 4 implantes por maxilar, aunque pueden ser necesarios 5 o 6 en ciertos casos del maxilar superior.

Uno de los puntos más atractivos de la técnica es que se pueden aplicar en un alto porcentaje de los casos con tasas de éxito superiores al 95\% (los implantes inferiores serán interforaminales incluso en situaciones de reabsorción extrema; en el maxilar superior se colocarán entre los senos maxilares disminuyendo la necesidad de regeneración que contraindicaría la técnica) $(2,3)$.

Además, se trata de una intervención en la que colocamos un menor número de implantes de lo habitual, con lo que facilitamos la higiene y, además, abaratamos los costes.

Centrándonos en el maxilar inferior, la filosofía del sistema se basa en:

- Uso de cuatro implantes en la zona mandibular anterior interforaminal.

- Angulación de los implantes posteriores con los ápices de los mismos hacia mesial, de manera que el punto de inserción pueda situarse en la vertical de los mentonianos o, incluso, un poco distal a ellos, a fin de disminuir el cantiléver distal de la futura prótesis.

\section{CASO CLÍNICO}

Paciente de sexo masculino de 54 años de Edad en ABEG, acude al Servicio del Postgrado de Rehabilitación Oral de la Facultad de Estomatología de la Universidad Peruana Cayetano Heredia. Al examen clínico intraoral presenta reborde alveolar inferior con reabsorción marcada del sector posterior, a nivel de zona de premolares presenta cuatro dientes en mal estado y extruidos con presencia de inflamación gingival leve generalizada, dificultad para la masticación debido a la falta de dientes postero -inferiores y plano oclusal alterado (Figura1). Al examen radiográfico se observa piezas dentarias 18, 34, 33, 43,44 con proporción coronoradicular desfavorable (Figura 2). Al paciente se le diagnostico enfermedad gingival inducida por placa dental y desorden funcional oclusal por ser edéntulo parcial superior e inferior, alteración de la guía anterior, alteración del plano oclusal, alteración de la dimensión vertical.

A la evaluación de las opciones de tratamiento propuestos y analizando costo-beneficio, paciente decidió por la exodoncias de dientes con la colocación de implantes oseointegrados y la rehabilitación implanto-soportada inferior y prótesis total superior convencional.

El tratamientos con implantes oseointegrados en el maxilar inferior tuvo como primera fase la exodoncias de las piezas dentarias $33,34,43,44$, con remodelado óseo y la colocación de cuatro implantes con instalación de una prótesis total superior e inferior transitoria restableciendo la dimensión vertical de la oclusión del paciente. Los implantes tuvieron una estabilidad primaria mínima de 60 N.cm y distribuidos en las posiciones: $32,34,42,44$,

El procedimiento quirúrgico se inició con la asepsia

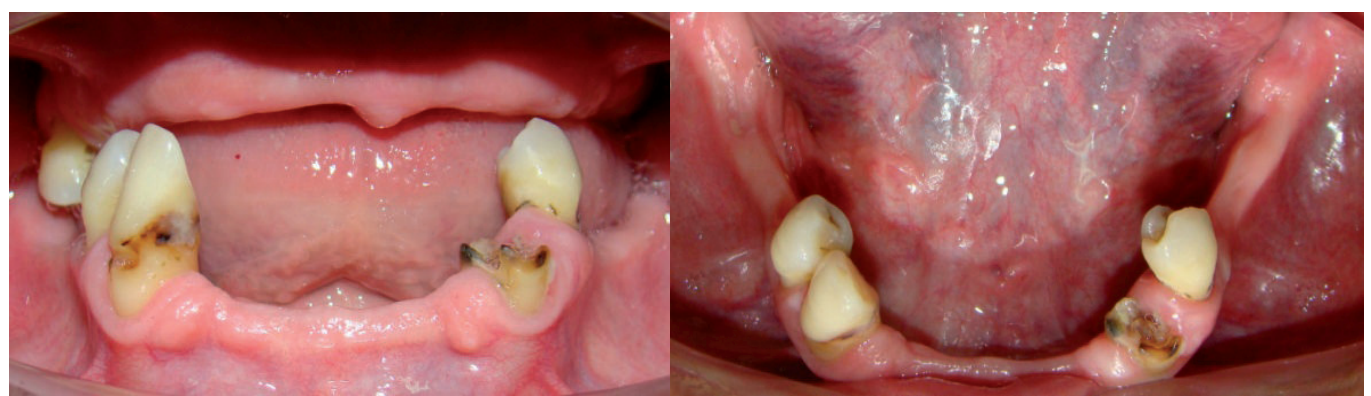

Figura 1. A y B. Fotografías intraorales de diagnóstico 


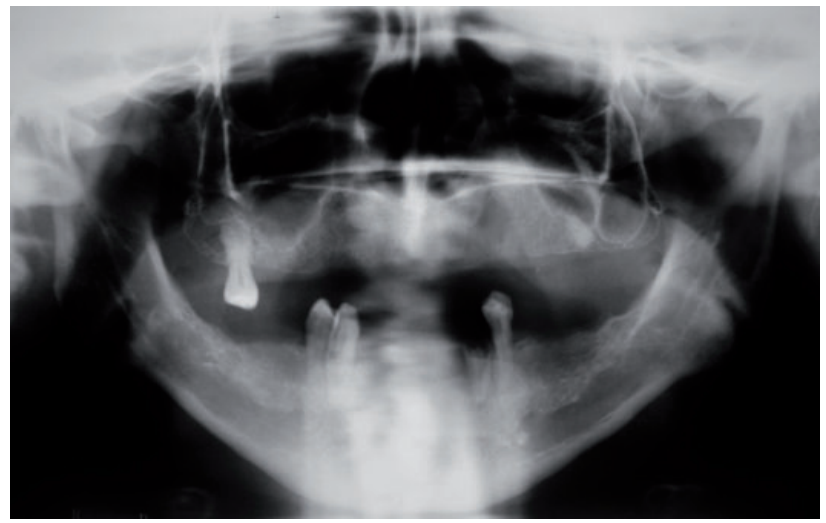

Figura 2. Radiografía panoramica

y antisepsia del campo operatorio, luego infiltración de anestesia local a nivel de nervios mentoneanos y fondo de surco vestibular de la zona anterior. Se realizó la incisión intrasurcal de la piezas 34,33,43 y 44 e incisión lineal supracrestal a nivel de las piezas $32-42$,se procede con el decolado mucoperióstico por vestibular, se visualiza ambas emergencias del nervio mentoniano, se procede a la exodoncia de las piezas $34,33,43$ y 44, curetaje y lavado de los alveolos con suero fisiológico. Luego se realiza el decolado mucoperióstico por lingual, se efectúo la osteoplastia del reborde óseo crestal con la sierra reciprocante con abundante refrigeración para crear una meseta, posteriormente con la ayuda de una fresa quirúrgica de baja velocidad se realizó un remodelado óseo final hasta conseguir una meseta de ancho mínimo de $5 \mathrm{~mm}$ (Figura 3 y 4). Se colocaron los implantes teniendo como referencia una guía quirúrgica, verificando la dirección e inclinación mediante los paralelizadores (Figura 5). Asi, fueron colocados 4 implantes cilíndricos de conexión externa con dimensiones

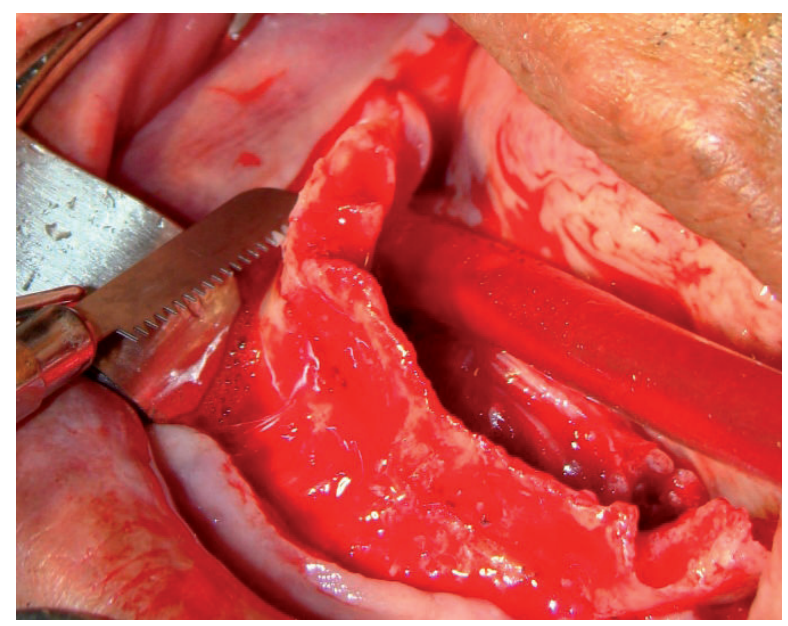

Figura 3. Osteoplastia del reborde óseo crestal con la sierra reciprocante

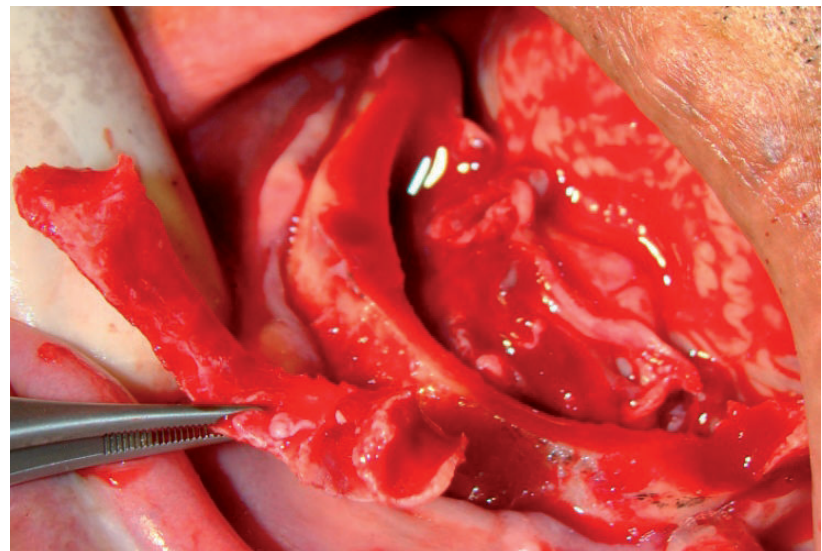

Figura 4. Remodelado óseo final hasta conseguir una meseta

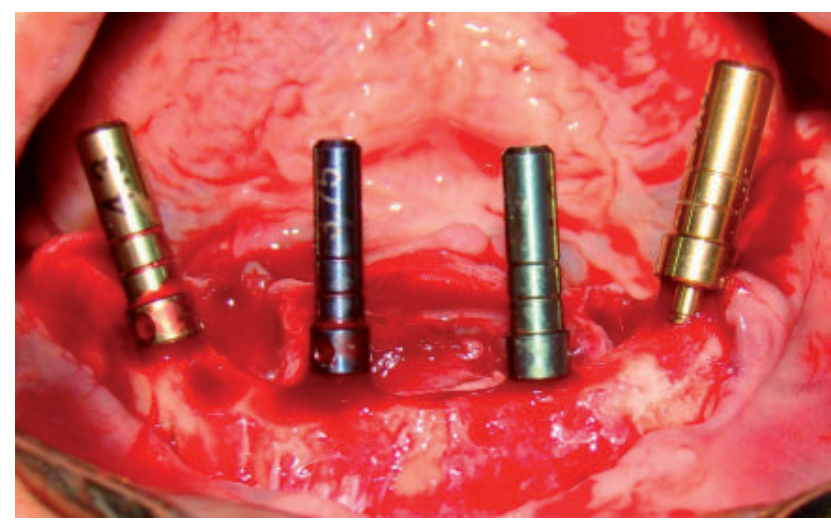

Figura 5. Verificación de la dirección e inclinación mediante los paralelizadores

de $3.75 \times 15 \mathrm{~mm}$, con un trabamiento inicial de 30 N.cm y final de 80 N.cm, los dos implantes distales se insertaron con 20 grados de angulación hacia distal aproximadamente. El procedimiento quirúrgico fue finalizado con la sutura del colgajo. Se indicó colocación de hielo externamente en el área operada durante las primeras 24 horas, antibióticos por 7 días y analgésicos por 3 días.

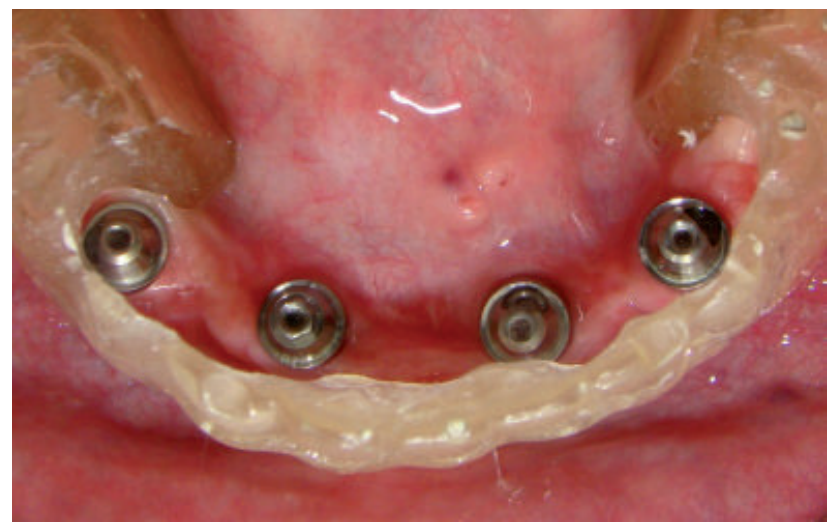

Figura 6. Prueba de pilares con la guía quirúrgica

Rev Estomatol Herediana. 2014 Ene-Mar;24(1) 
Luego de dos meses de oseointegración de los implantes, se procedió a la segunda fase quirúrgica donde se probaron los pilares para verificar la angulación, paralelismo y que exista espacio suficiente con respecto a los dientes antero-inferiores para lo cual nos ayudamos de la guía quirúrgica, que sirve como guía multifuncional (Figura 6). Posteriormente los pilares intermediarios de tipo mini pilar cónico SF recto fueron instalados en los implantes a nivel de las piezas 32,42 y recibieron un torque de 32 N.cm, para los implantes distales a nivel de las piezas 34,44 se utilizaron pilares intermediarios de tipo mini pilar cónico angulado de 17 grados con un toque de 20 N.cm (Figura 7). Se procedió a la transferencia con la ayuda de una cubeta abierta y silicona por adición. Se realizó el vaciado con yeso extra duro .Se confecciono una placa base rodete sobre dos tranfers

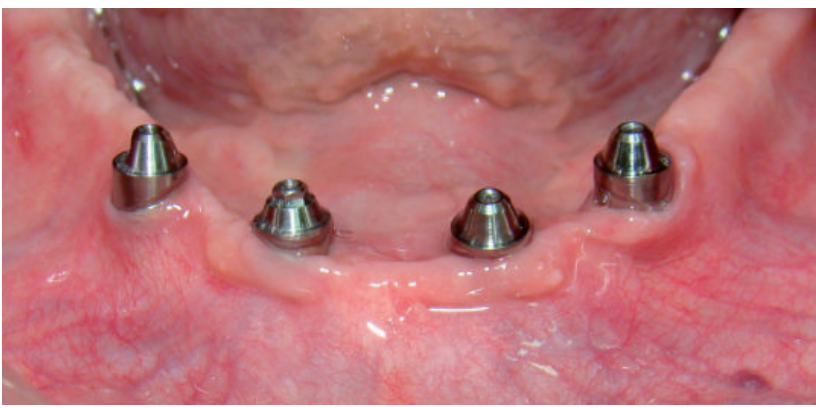

Figura 7. Instalación de pilares definitivos

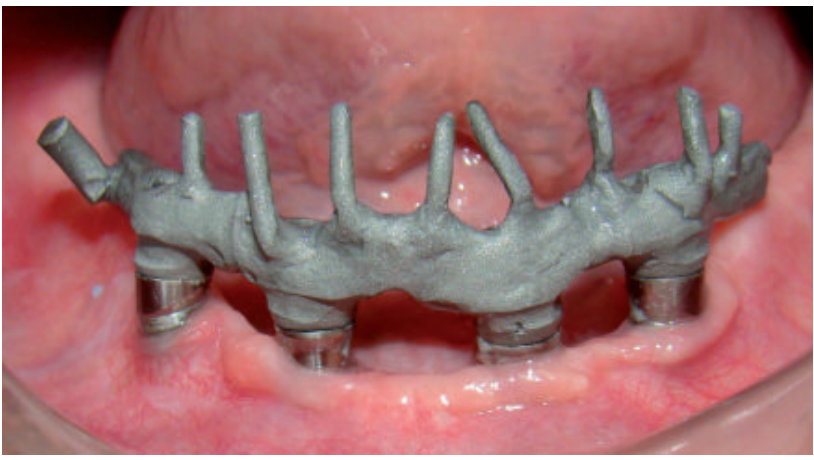

Figura 8. Prueba de supra-estructura donde se observa el asentamiento pasivo.

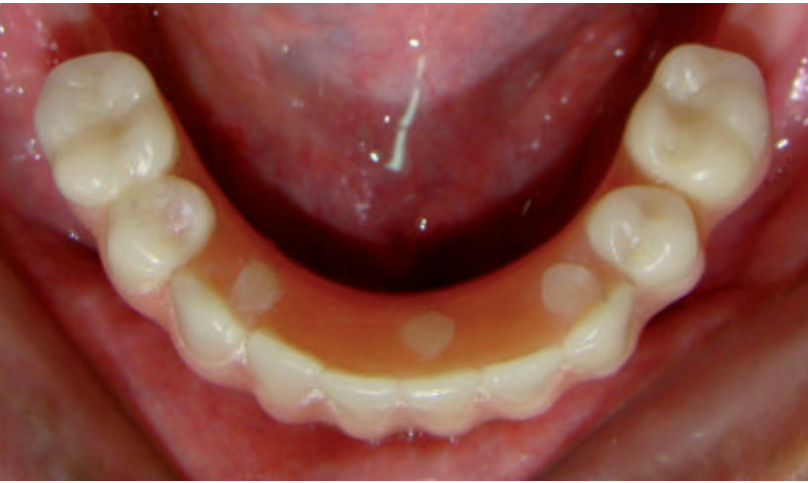

Figura 9. Los orificios de acceso fueron cubiertos con resina fotopolimerizable.

con los cuales se evaluó el soporte labial, contorno y la dimensión vertical. Se realizó la prueba de enfilado donde se evaluó estética y fonética. Posteriormente, el laboratorio confecciono una supra-estructura de $\mathrm{Cr} \mathrm{Ni}$ con los cilindros calcinables del mini pilar cónico (Neodent, Curitiba, Brasil). Se probó la estructura en boca y se verifico el asentamiento y pasividad de forma visual y radiográfica (Figura 8). Luego el laboratorio realizo la reposición del enfilado sobre la supra-estructura metálica esta se probó en boca verificando nuevamente la dimensión vertical, soporte labial, estética, fonética y la oclusión. Luego se procedió al procesado de la prótesis.

Para la instalación se procedió al retiro de las tapas de protección, la prótesis fue colocada en posición y se evaluó la adaptación. Estando en condiciones adecuadas, fue instalada empleando los tornillos protésicos torqueados a 10 N.cm. Los orificios de acceso fueron cubiertos con cinta teflón y resina fotopolimerizable (Figura 9). La oclusión fue verificada garantizando contactos céntricos en los dientes posteriores (Figura 10). Al paciente se le brindo indicaciones respecto a la alimentación e higiene.

Además en tomas radiográficas posteriores fue posible constatar una correcta adaptación de los
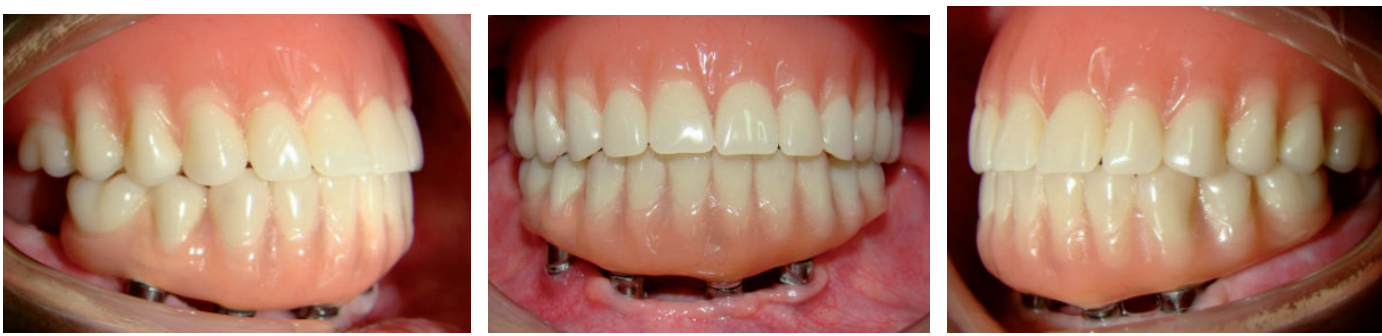

Figura 10. Instalación de prótesis hibrida inferior y prótesis total superior 
componentes protésicos sobre los pilares de los implantes.

\section{DISCUSIÓN}

Se han reportado en la literatura las ventajas del tratamiento del edentulismo maxilar y mandibular con prótesis implanto-soportada devolviendo la función y estética, lo que les permite a los pacientes mejorar su condición social y psicológica $(4,5)$.

Se debe prestar especial atención a la fase de diagnóstico. Independientemente de la técnica utilizada, fase quirúrgica, aspectos oclusales y estéticas, así como las expectativas del paciente (6). Las contraindicaciones de los implantes inmediatos son la presencia de infección activa, hueso insuficiente $(<3 \mathrm{~mm})$, fenestración cervical en la pared vestibular o una destrucción del lecho alveolar (7).

Pinto y col. (8) Realizaron reportes de casos clínicos donde concluyeron que debido al alto índice de éxito de los implantes oseointegrados se creó la expectativa de utilizarlos para remplazar los dientes con pronóstico malo o reservado para lo cual se debe de realizar un diagnóstico integral y la aplicación de técnicas basadas y sustentadas por una evidencia científica. Proponen que se debe presentar diferentes alternativas de tratamientos al paciente explicándole las ventajas y desventajas de cada opción en relación a los aspectos biológicos y financieros donde el paciente escoja una de las posibilidades presentadas. Esta información debe ser anexada en la historia clínica por razones legales.

Análisis biomecánico de la rehabilitación implanto-soportada convencional (2 etapas), revela que el estrés que sufre el implante como resultado de la desadaptación de la prótesis puede estar presente después de muchos años de la oseointegración (9). Por esta razón, la desadaptación puede conducir a problemas tales como el aflojamiento del tornillo, la fractura de prótesis o implantes y pérdida de hueso (10). Por lo tanto, un ajuste preciso entre el pilar del implante y de la supra-estructura dan como resultado ausencia de estrés, estos factores son importantes para el éxito a largo plazo de las restauraciones implantosoportadas.

En el año 1997 Tarnow y col.(11) dan a conocer los resultados de un estudio de 107 implantes Nobel,
ITI, 3i y Astra (69 sometidos a carga inmediata y 38 diferida ) colocados sobre 10 pacientes totalmente edéntulos ( 6 de ellos intervenidos en mandíbula y 4 en el maxilar). Ponían un mínimo de 10 fijaciones de al menos $10 \mathrm{~mm}$ por arcada, dejando al menos 5 de ellos sumergidos por motivo médico-legal; el resto se cargaba con una prótesis fija provisional el mismo día de la cirugía. Tras un seguimiento de 12 a 60 meses obtuvieron un éxito del $97,1 \%$ en los de carga inmediata y un $97,4 \%$ en los de carga diferida.

También en 1997, Balshi y Wolfinger (12) publicaron un estudio sobre 13 pacientes en el que colocaron 10 implantes Bränemark de al menos $7 \mathrm{~mm}$ de altura en la mandíbula. De estos implantes, 40 (4 por paciente) se cargaron tras la cirugía para soportar una prótesis transicional fija. Con este protocolo tan sólo se consiguió un $80 \%$ de éxito, mientras que esta tasa se eleva al 95,6\% en los cargados según protocolo convencional.

Paulo Maló y col.(2) introdujeron en el 2003 el concepto «All-on-Four» con implantes Bränemark de carga inmediata. Este protocolo consiste en la colocación de 4 implantes intermentonianos de al menos $10 \mathrm{~mm}$, los dos anteriores en la dirección del hueso y los dos posteriores con una inclinación de unos $30^{\circ}$, emergiendo a nivel del segundo premolar. La prótesis híbrida que soportan los implantes, previa colocación de pilares angulados en las fijaciones posteriores, reponen la arcada hasta el primer molar, y eran colocadas antes de dos horas tras la cirugía. En el trabajo publicado, sobre 44 pacientes, colocaron 176 implantes de carga inmediata (de los cuales 45 eran inmediatos) y otros 62 implantes de rescate. Tras un seguimiento entre 6 meses y 2 años, lograron un éxito del $96,7 \%$ y un $100 \%$ de la prótesis.

Según los trabajos revisados, el tipo de prótesis juega un papel importante en los resultados de los implantes cargados de forma inmediata. Ampliamente demostrado está el éxito de las sobredentaduras y prótesis híbridas inferiores, con resultados similares a los de la carga según protocolos convencionales (13-15).

\section{CONCLUSIONES}

Se ha demostrado que las prótesis implanto soportadas son una excelente opción para la rehabilitación de pacientes edéntulos totales, ya que 
les devuelve la función y la estética, lo que les permite mejorar su condición social y psicológica. También se ha observado que este tipo de prótesis proporciona muchas ventajas, entre estas ventajas se pueden citar: menor pérdida ósea, acceso mejorado para la higiene oral y más espacio para corregir discrepancias en la relación del arco dental y además mejora la retención y la estabilidad de la prótesis.

Está ampliamente demostrado que los resultados obtenidos en rehabilitaciones completas inferiores con implantes de carga diferida, ya sea mediante sobredentaduras, híbridas o prótesis fijas tipo puente son similares a los conseguidos mediante protocolo de carga inmediata.

\section{CORRESPONDENCIA}

Dulio Rafael Maravi Escurra

Calle francisca Sánchez de Pagador Urb. Pando Lima 34, Perú.

Teléfono: 945995942

e-mail: dulio_maravi@yahoo.es

\section{REFERENCIAS BIBLIOGRÁFICAS}

1. Branemark P, Zarb T, Albrektsson G. Prótesis-tejidointegradas. Barcelona: Edit quintessence; 1999.

2. Malo P, Rangert B, Nobre M. «All-on-Four» immediate-function concept with Brånemark System implants for completely edentulous mandibles: a retrospective clinical study. Clin Implant Dent Relat Res. 2003;5(1):2-9.

3. Malo P, Rangert B, Nobre M. «All-on-Four» immediatefunction concept with Brånemark System implants for completely edentulous maxillae: a 1-year retrospective clinical study. Clin Implant Dent Relat Res. 2005;7(1): 88-94.

4. Tarnow DP, Emtiaz S, Classi A. Immediate loading of threaded implants at stage 1 surgery in edentulous arches: Ten consecutive case reports with 1- to 5-year data. Int J Oral Maxillofac Implants. 1997 MayJun;12(3):319-24.

5. Schnitman PA, Wohrle PS, Rubenstein JE, DaSilva JD, Wang NH. Ten-year results for Brånemark implants immediately loaded with fixed prostheses at implant placement. Int J Oral Maxillofac Implants. 1997 Jul-
Aug;12(4):495-503.

6. Gallucci GO, Bernard JP, Bertosa M, Belser UC. Immediate loading with fixed screw-retained provisional restorations in edentulous jaws: The pickup technique. Int J Oral Maxillofac Implants. 2004 JulAug;19(4):524-33.

7. Schropp L, Wenzel A, Kostopoulos L, Karring T. Bone healing and soft tissue contour changes following single-tooth extraction: a clinical and radiographic 12-month prospective study. Int $\mathrm{J}$ Periodontics Restorative Dent. 2003;23(4):313-23

8. Pinto AVS, de Sousa FL, Bellinghausen Neto AE, Anabuki N, Boniati D, Pinto MS. El dilema de la substitución de dientes naturales por implantes oseointegrados: relato de dos casos clínicos. Rev Implant news. 2010;1(1):13-20

9. Jemt T, Book K. Prosthesis misfit and marginal bone loss in edentulous implant patients. Int J Oral Maxillofac Implants. 1996 Sep-Oct;11(5):620-5.

10. Burguete RL, Johns RB, King T, Patterson EA. Tightening characteristics for screwed joints in osseointegrated dental implants. J Prosthet Dent. 1994 Jun;71(6):592-9.

11. Tarnow DP, Emtiaz S, Classi A. Immediate loading of threaded implants at stage 1 surgery in edentulous arches: Ten consecutive case reports with 1 to 5 years data. Int J Oral Maxillofac Implants. 1997 MayJun;12(3):319-24.

12. Balshi TJ, Wolfinger GJ. Immediate loading of Brånemark implants in edentulous mandibles: A preliminary report. Implant Dent. 1997; 6:83-8.

13. Wolfinger GJ, Balshi TJ, Rangert B. Immediate functional loading of Branemark system implants in edentulous mandibles: clinical report of the results of developmental and simplified protocols. Int J Oral Maxillofac Implants. 2003 Mar-Apr;18(2):250-7.

14. Attard NJ, Zarb GA. Immediate and early implant loading protocols: a literature review of clinical studies. J Prosthet Dent. 2005; 94:242-58.

15. Horiuchi K, Hiroya U, Yamamoto K, Sugimura M. Immediate loading of Branemark System implants following placement in edentulous patients: a clinical report. Int J Oral Maxillofac Implants. 2000 NovDec;15(6):824-30.

Recibido : $12 / 08 / 2013$

Aceptado: 04/10/2013 